\title{
Efecto de diferentes niveles de riego en el rendimiento de rosa mosqueta (Rosa canina L.), en la zona Centro-Sur de Chile
}

\author{
Effect of different irrigation levels on the yield of rose hip (Rosa canina L.), \\ in South-Central Chile
}

\author{
Quezada, C. ${ }^{a^{*}}$, Sandoval, M. ${ }^{a}$, Serri, H. ${ }^{b}$, Barra, L. ${ }^{a}$, Rillon, S. ${ }^{c}$, Barriga, R. ${ }^{c}$ \\ ${ }^{a}$ Departamento de Suelos y Recursos Naturales, Facultad de Agronomía, \\ Universidad de Concepción, Casilla 537, Chillán. \\ ${ }^{b}$ Departamento de Producción Vegetal, Facultad de Agronomía, \\ Universidad de Concepción, Casilla 537, Chillán. \\ ${ }^{c}$ Sociedad Agrícola y Forestal Casino Ltda., Chillán Viejo.
}

\begin{tabular}{l} 
A R T I C L I N F O \\
\hline Article history: \\
Received 11.09.14 \\
Accepted 11.12 .14 \\
\hline Keywords: \\
Rainfed \\
Stomatal conductance \\
Dry pulp \\
Water productivity \\
\hline Original Research Article, \\
Soil Science \\
\hline${ }^{*}$ Corresponding author: \\
Celerino Quezada \\
E-mail address: \\
cequezad@udec.cl
\end{tabular}

\begin{abstract}
A B S T R A C T
The water resource is the limiting factor in rainfed areas for the introduction of new production alternatives. The objective of this field experiment was to evaluate the effect of different water levels on yield components of rose hip orchards (Rosa canina L.) located in South-Central Chile, during the 2011-2012 growing season, in an Aquic Palexeralfs soil under drip irrigation. The field experimental design was completely randomized with four water treatments set as a function of applied irrigation depth (ID) in the farm (ID) : 0,25 ID; 0,5 ID; 1 ID (control) and 2 ID with three replicates of five plants. Applied water volume, soil water content, stomatal conductance, yield components, industrial yield and water productivity were determined. Results showed that the water requirements of rose hip were lower and equivalent to supplementary irrigation during the period of maximum evapotranspiration. The different water levels did not affect the physiological parameters and yield components. Application of the 50\% irrigation depth achieved a good dry pulp yield and it is an adequate irrigation strategy for rainfed areas with water scarcity.
\end{abstract}

\section{RESUMEN}

El recurso hídrico es el factor limitante en zonas de secano para introducir nuevas alternativas productivas. El objetivo de esta investigación fue evaluar el efecto de diferentes niveles de riego en el rendimiento de Rosa Mosqueta (Rosa canina L.) en un suelo Aquic Palexeralfs bajo riego por goteo en la zona centro-sur de Chile. El diseño experimental fue completamente al azar con cuatro tratamientos hídricos en función de la lámina de riego aplicada en el predio (LR): 0,25 LR; 0,5 LR; 1 LR y 2 LR, con tres repeticiones de 5 plantas. Se evaluó volumen de agua aplicada, contenido de agua en el suelo, conductancia estomática, componentes de rendimiento, rendimiento industrial y productividad del agua. Los resultados obtenidos muestran que los requerimientos hídricos de Rosa Mosqueta fueron bajos y equivalentes a un riego suplementario en el período de mayor evapotranspiración. Los diferentes niveles de aplicación de agua no afectaron significativamente los parámetros fisiológicos ni los componentes de rendimiento. La aplicación del 50\% de la lámina de riego presentó un buen rendimiento de pulpa seca y es una estrategia de riego adecuada para zonas de secano con escasez del recurso hídrico.

Palabras clave: Secano, conductancia estomática, pulpa seca, productividad del agua.

\section{INTRODUCCIÓN}

Rosa Mosqueta crece en la zona central de Chile en áreas entre $36^{\circ} \mathrm{S}$ y $38^{\circ} \mathrm{S}$ donde se han descrito tres especies : Rosa canina L., Rosa rubiginosa L., y Rosa moschata Herm., que han sido clasificadas como nuevas especies nativas (Joublan y Ríos, 2005). Además, en la región del Bío Bío se han logrado detectar siete variedades, ecotipos que se diferencian entre sí por el tamaño, forma y color del fruto, época de maduración y hábitos de crecimiento, factores de gran importancia en el rendimiento de pulpa seca (Sudzuki, 1997).

La adaptación de esta planta a las condiciones agroecológicas se desarrolla en forma silvestre o natural, entre las regiones del Maule y de la Araucanía, en zonas con suelos erosionados de secano, y con escasas alternativas de cultivos agrícolas rentables (Berti y Joublan, 1997) y cubre aproximadamente unas 15.000 
hectáreas en forma silvestre en el país, siendo en la región del Bío Bío donde se concentra la mayor parte de esa superficie (Sudzuki, 1997). Por su parte, Medel (1979) destaca que es uno de los arbustos frutales con mayores posibilidades de desarrollo, principalmente en el sur de Chile por las características agroecológicas apropiadas, manejo cultural simple, mayor incorporación de trabajo y amplias posibilidades de mercado interno y externo para productos con diferentes grados de industrialización.

La importancia económica de la Rosa Mosqueta radica en su uso con fines agroindustriales para mermeladas, jalea, jugos, fabricación de tintas y aceites esenciales, siendo éste último la principal propiedad que posee por su alto poder regenerativo de tejidos (Sudzuki, 1997). Además, presenta altos contenidos de vitamina C (200-1000 mg/100 g) y carbohidratos (5,86 - 24,52 g/100 g (Kovacs et al., 2005).

En el país el cultivo se ha desarrollado por iniciativa de empresas privadas, que con el apoyo de organismos públicos, han logrado una mayor tecnificación del manejo agronómico. Los frutos son cosechados por recolectores y vendidos a la agroindustria donde son secados, limpiados y luego exportados a países en Europa, principalmente Alemania, pero la recolección silvestre es causa de abastecimiento irregular y baja calidad del producto (Joublan y Ríos, 2005). La demanda creciente de sub-productos como mermeladas, jugos, té, y postres (Uggla y Martinsson, 2005) genera un mayor interés por evaluar genotipos seleccionados de poblaciones silvestres (Günes, 2010).

El riego es una de las tecnologías a desarrollar en el cultivo de la Rosa Mosqueta, ya que en zonas de secano la precipitación es baja, la evapotranspiración es alta y los períodos de sequía son frecuentes y es necesario garantizar el uso eficiente del agua (Vita et al., 2004; Alarcón et al., 2006). Al respecto, Oweis et al. (2000) señalan que la eficiencia de uso del agua (EUA) puede ser mejorada aplicando riego suplementario para satisfacer los 2/3 de los requerimientos hídricos del cultivo, utilizando sistemas de riego tecnificado, cosecha de aguas lluvias (Billib et al., 2009) y fuentes alternativas de agua como vertientes y norias.

El sistema de riego localizado permite mantener niveles de humedad óptimos en la zona radical, favoreciendo el uso agrícola de zonas de secano donde existen suelos marginales de bajo valor agrícola. Sin embargo, no existe información sobre el requerimiento hídrico de la Rosa Mosqueta, ni del efecto de diferentes niveles de reposición hídrica, sobre la producción y rendimiento industrial. Al respecto, Schmidt (1992), en frambuesa determina que los niveles de reposición hídrica varían entre 88 y $103 \%$ de la evaporación de bandeja para plantas de dos años en la IX región. Por su parte, Obando y Mc Leod (2009), en zarzaparrillas establecieron que una dosis por planta de 4 litros de agua al día, ase- gura buen crecimiento, alta producción, frutos grandes y uniformes. En cambio, Nissen et al. (2010) obtuvieron los mejores rendimiento en frutos de murtilla en la zona de Valdivia con la aplicación del 40\% de la evaporación de bandeja, pero con un aporte de lluvias $(775,2 \mathrm{~mm})$ y una aplicación total de $943 \mathrm{~mm}$ (6 riegos).

El objetivo general de esta investigación fue determinar los requerimientos hídricos y evaluar el efecto de diferentes niveles de riego sobre componentes de rendimiento y calidad industrial en un huerto experimental de Rosa Mosqueta (Rosa canina L.) en la zona centro-sur de Chile.

\section{MATERIALES Y MÉTODOS}

\section{Sitio experimental}

El ensayo se realizó durante la temporada 2011 2012 en el Huerto Experimental Santa Adriana perteneciente a la Sociedad Agrícola y Forestal Casino Ltda., ubicado en la Panamericana Sur a 5 km de Chillán, provincia de Ñuble, Región del Bío Bío, Chile (36³7' Sur y $72^{\circ} 10^{\prime}$ Oeste, 97 m.s.n.m).

El suelo corresponde a un Alfisol, Serie Mirador (Aquic Palexeralfs) cuyo material de origen es toba volcánica andesítico-basáltica, con profundidad entre 50 y $90 \mathrm{~cm}$, y topografía plana a suavemente ondulada. Presenta textura franco arcillosa y color pardo rojizo oscuro en la superficie a textura arcillosa y color pardo rojizo oscuro a pardo rojizo en profundidad, concreciones ferro-manganésicas escasas en superficie a comunes en profundidad. El suelo posee una permeabilidad moderadamente lenta y drenaje moderado a imperfecto (Stolpe, 2006).

El clima es Mediterráneo templado perteneciente al Agroclima Chillán que se caracteriza por una temperatura media anual de $14^{\circ} \mathrm{C}$, una temperatura mínima promedio del mes más frío (Julio) entre 3,5 y $5^{\circ} \mathrm{C}$ una temperatura máxima promedio (Enero) $29^{\circ} \mathrm{C}$, siendo las sumas térmicas base $5^{\circ} \mathrm{C}$ de 3.106 grados días. El período libre de heladas es de 5 meses (Noviembre a Marzo), y las horas de frío acumuladas desde Marzo a Diciembre suman 1.400 horas (Del Pozo y Del Canto, 1999). La precipitación anual es de $1.006 \mathrm{~mm}$ y la evaporación de bandeja (Eb) anual de $1.257 \mathrm{~mm}$, en la temporada de riego (Octubre a Marzo) la Eb alcanza a $1.079 \mathrm{~mm}$ (Cuadro 1).

\section{Manejo del huerto}

El establecimiento del huerto de Rosa Mosqueta se realizó en el mes de Noviembre del año 2009, con un marco de plantación de 3 x 1 m (3.333 plantas ha-1). En relación a labores culturales, se realizó poda manual, la cual tiene como objetivo la limpieza basal de la planta y una fertilización con $20 \mathrm{~g}$ de urea por planta. Las male- 
Cuadro 1. Precipitación y evaporación de bandeja en la zona de Chillán, Chile (1993 - 2012).

Table 1. Rainfall and pan evaporation in the Chillán area, Chile (1993-2012).

\begin{tabular}{lcc}
\hline Mes & $\begin{array}{c}\text { Precipitación } \\
(\mathrm{mm})\end{array}$ & $\begin{array}{c}\text { Evaporación de } \\
\text { bandeja (mm) }\end{array}$ \\
\hline Enero & 9 & 256 \\
Febrero & 31 & 193 \\
Marzo & 17 & 148 \\
Abril & 67 & 71 \\
Mayo & 155 & 20 \\
Junio & 234 & 6 \\
Julio & 168 & 9 \\
Agosto & 136 & 21 \\
Septiembre & 72 & 51 \\
Octubre & 62 & 101 \\
Noviembre & 32 & 158 \\
Diciembre & 23 & 223 \\
\hline
\end{tabular}

Fuente: Universidad de Concepción, Facultad de Ingeniería Agrícola, Departamento de Recursos Hídricos, Campus Chillán.

zas son controladas en forma mecánica con desbrozadora y segadora, y no se realiza control fitosanitario. El método de riego es por goteo, con emisores integrales auto-compensados Netafim, caudal $4 \mathrm{~L} \mathrm{~h}^{-1}$ y una presión nominal de 10 m.c.a., a una distancia de $1 \mathrm{~m}$ sobre la línea de riego de $62 \mathrm{~m}$. de largo. La programación de riego considera una frecuencia de riego de 7 días y tiempo de riego de 2 horas con un gotero por planta de $4 \mathrm{~L} \mathrm{~h}^{-1}$. La fuente de agua para riego es un pozo profundo de $50 \mathrm{~m}$. Es impulsada y distribuida en el huerto mediante una bomba sumergible marca Franklin Electric de 7,5 HP, con un diámetro de 2" de salida y caudal máximo de $15 \mathrm{~m}^{3} \mathrm{~h}^{-1}$.

\section{Diseño experimental}

El ensayo se realizó en 4 laterales del huerto aplicando un diseño de bloques completamente al azar con cuatro tratamientos hídricos y tres repeticiones de 5 plantas, estableciendo una separación de 3 plantas entre tratamientos para evitar el efecto borde. Los tratamientos hídricos (T) corresponden a diferentes niveles de aplicación del agua en función de la lámina de riego aplicada en el predio (LR) que utiliza un gotero por planta de $4 \mathrm{~L} \mathrm{~h}^{-1}$. Estos fueron, T1: 0,25 LR con goteros de $1 \mathrm{~L} \mathrm{~h}^{-1}$,T2: 0,5 LR con goteros de $2 \mathrm{~L} \mathrm{~h}^{-1}$; T3: $1 \mathrm{LR}$ con goteros de $4 \mathrm{~L} \mathrm{~h}^{-1}$ y T4: $2 \mathrm{LR}$ con goteros de $8 \mathrm{~L} \mathrm{~h}^{-1}$. El riego se realizó entre el 21 de Diciembre 2011 y el 14 de Febrero del 2012 de acuerdo a la programación de la Empresa.

\section{Evaluaciones}

\section{Volumen de agua aplicado}

El volumen aplicado por tratamiento se determinó aforando los goteros cada 15 días por el método volumétrico, aplicando la siguiente relación:

$$
\text { Vap }=[\text { qe } \times \text { Ne } \times \operatorname{Tr} \times \mathrm{Nr} \times \mathrm{Npl}] / 1000\left[\mathrm{~m}^{3} \mathrm{ha}^{-1}\right]
$$

donde: Vap es el volumen de agua aplicado $\left(\mathrm{m}^{3} \mathrm{ha}^{-1}\right)$, qe es el caudal del emisor $\left(\mathrm{L} \mathrm{h}^{-1}\right)$, Ne es el número de emisores por planta, $\mathrm{Tr}$ el tiempo de riego (h), $\mathrm{Nr}$ es el número de riegos y $\mathrm{Npl}$ el número de plantas por hectárea y 1000 es el factor de transformación de litros a $\mathrm{m}^{3}$..

\section{Características físico hídricas del suelo}

Las propiedades físico-hídricas del suelo se determinaron por horizonte para las profundidades de 0-28, 28-64 y 64-100 cm (Cuadro 2) en una calicata con dimensiones de $1,0 \times 0,7 \times 1,2 \mathrm{~m}$. Las muestras fueron analizadas en el Laboratorio de Física de Suelos de la Facultad de Agronomía de la Universidad de Concepción. La textura fue determinada por el método del hidrómetro de Bouyoucos mejorado, el cual relaciona los resultados granulométricos con el triángulo textural USDA, donde la interpolación de los datos definen la clase textural (Sandoval et al., 2012). La Capacidad de Campo (CC) y Punto de Marchitez Permanente (PMP), fueron determinados por el método de la olla a presión a tensiones de $\left(-33 \mathrm{~J} \mathrm{~kg}^{-1}\right)$ y $\left(-1500 \mathrm{~J} \mathrm{~kg}^{-1}\right)$, respectivamente. La densidad aparente (Da), fue determinada en tres muestras por horizonte por el método del cilindro con dimensiones de 3,5 x $5 \mathrm{~cm}$ ( (Sandoval et al., 2012).

\section{Contenido de agua del suelo}

El contenido de agua del suelo fue determinado por el método gravimétrico y expresado como contenido volumétrico de agua multiplicado el contenido de agua gravimétrico por la densidad aparente del suelo y dividida por la densidad del agua (Varble y Chávez, 2011). El muestreo de suelos se realizó con barreno agrológico por tratamiento y repetición, una vez a la semana, a $20 \mathrm{~cm}$ de distancia de la planta y a $30 \mathrm{~cm}$ de profundidad, zona de mayor concentración de raíces. Las muestras fueron sometidas a una temperatura de $105^{\circ} \mathrm{C}$ por 48 horas en un horno de secado, período de tiempo en el cual se elimina toda el agua del suelo y se obtiene peso seco constante.

\section{Conductancia estomática}

La conductancia estomática $\left[\mathrm{mmol} \mathrm{m}^{-2} \mathrm{~s}^{-1}\right]$ se midió con un porómetro de difusión (AP-4 Delta T Devices, Cambridge, UK) una vez a la semana, durante las horas de 
Cuadro 2. Características físico-hídricas del suelo Aquic Palexeralfs en el sitio experimental.

Table 2. Physical-hydric characteristics of an Aquic Palexeralfs soil in the experimental site.

\begin{tabular}{ccccccccc}
\hline $\begin{array}{c}\text { Prof. } \\
{[\mathrm{cm}]}\end{array}$ & $\begin{array}{c}\text { Arcilla } \\
{[\%]}\end{array}$ & $\begin{array}{c}\text { Limo } \\
{[\%]}\end{array}$ & $\begin{array}{c}\text { Arena } \\
{[\%]}\end{array}$ & $\begin{array}{c}\text { Clase } \\
\text { textural }\end{array}$ & $\begin{array}{c}\mathrm{Da} \\
{\left[\mathrm{g} \mathrm{cm}^{-3}\right]}\end{array}$ & $\begin{array}{c}\theta_{\mathrm{cc}} \\
{\left[\mathrm{m}^{3} \mathrm{~m}^{-3}\right]}\end{array}$ & $\begin{array}{c}\theta_{\text {pmp }} \\
{\left[\mathrm{m}^{3} \mathrm{~m}^{-3}\right]}\end{array}$ & $\begin{array}{c}\mathrm{HA} \\
{\left[\mathrm{m}^{3} \mathrm{~m}^{-3}\right]}\end{array}$ \\
\hline $0-28$ & 39,0 & 36,9 & 24,1 & $\begin{array}{c}\text { Franco } \\
\text { arcillosa }\end{array}$ & 1,25 & 0,37 & 0,19 & 0,18 \\
$28-64$ & 37,0 & 30,7 & 32,3 & $\begin{array}{c}\text { Franco } \\
\text { arcillosa }\end{array}$ & 1,40 & 0,39 & 0,20 & 0,19 \\
$64-100$ & 36,9 & 32,8 & 30,3 & $\begin{array}{c}\text { Franco } \\
\text { arcillosa }\end{array}$ & 1,35 & 0,37 & 0,19 & 0,18 \\
\hline
\end{tabular}

Da: Densidad aparente; CC: Capacidad de campo; PMP: Punto de marchitez permanente; HA: Humedad Aprovechable $\left(\left[\theta_{c c}-\theta_{\text {pmp }}\right]\right) ;{ }^{1}$ Sistema USDA.

mayor radiación solar del día [12:00 - 13:30 hr]. En la zona de mayor exposición al sol de la planta, se eligió una hoja verde, sana y sin daño mecánico en la partes: superior, media y baja por cada tratamiento y repetición (Quezada et al., 2005).

\section{Componentes del rendimiento}

Los componentes del rendimiento se evaluaron en los tres arbustos centrales de cada repetición y fueron los siguientes:

\section{Número de frutos por planta}

El número de frutos por planta se determinó contando su cantidad de forma separada para las tres plantas de cada repetición.

\section{Peso promedio de frutos}

El peso promedio de frutos se evaluó seleccionando 100 frutos al azar por planta, los que fueron pesados en una balanza digital Snowrex para 1.200 g con sensibilidad de $1 \mathrm{~g}$.

\section{Diámetro longitudinal y ecuatorial}

El diámetro longitudinal y ecuatorial se midió en 100 frutos seleccionados al azar con un calibrador digital CE para $150 \mathrm{~mm}$ con sensibilidad de $1 \mathrm{~mm}$.

\section{Rendimiento}

El rendimiento se obtuvo pesando en una balanza digital Mettler Toledo para $30 \mathrm{~kg}$ con sensibilidad de 5 $\mathrm{g}$, todos los frutos producidos por cada planta de la repetición, para luego promediarlos y obtener la pro- ducción promedio por planta en cada tratamiento. El rendimiento en $\mathrm{kg} \mathrm{ha}^{-1}$ se calculó multiplicando la densidad de plantación por la producción por planta.

\section{Productividad del agua}

La productividad del agua (PA) fue calculada mediante la relación entre el peso de los frutos de Rosa Mosqueta $(\mathrm{kg})$ y el volumen de agua aplicada $\left(\mathrm{m}^{3}\right)$ tanto para el agua total aplicada (riego + lluvia) y como para el riego suplementario. Además, se calculó la relación entre diferencial de rendimiento y el de agua aplicada entre tratamientos, con el fin de determinar la función de cantidad de agua versus producción.

\section{Rendimiento Industrial}

El rendimiento industrial se expresó como peso de fruto fresco necesario para obtener un kilo de pulpa seca y se obtuvo cosechando una muestra de $500 \mathrm{~g}$ de fruto fresco la cual fue deshidratada para obtener el peso de pulpa seca, y luego se realizó la transformación a kilo por kilo. La muestra fue deshidratada en túnel de secado hasta un $8 \%$ de humedad, luego se rompió el fruto para sacar las semillas y pelusas, dejando la pulpa seca limpia de impurezas. Este análisis se realizó en el Laboratorio perteneciente a la Sociedad Agrícola y Forestal Casino Ltda.

\section{Análisis estadístico}

Los resultados obtenidos fueron sometidos a un análisis de varianza (ANDEVA) y a un test de comparación de medias mediante la prueba de Tukey con un nivel de significancia de un 5\% (Infostat, 2004). Además los datos fueron normalizados mediante el test de ShapiroWilks. 


\section{RESULTADOS Y DISCUSIÓN}

\section{Agua aplicada}

Los volúmenes de agua de riego aplicados durante la temporada comprendida entre el 21 de Diciembre del 2011 y el 14 de Febrero del 2012 variaron entre $87 \mathrm{~m}^{3}$ ha ${ }^{-1}$ para $\mathrm{T} 1$ y $673 \mathrm{~m}^{3} \mathrm{ha}^{-1}$ para $\mathrm{T} 4$, estimados como muy bajos en comparación a frambuesa con $3.000 \mathrm{~m}^{3} \mathrm{ha}^{-1}$ (Holzapfel et al., 1994). Considerando el aporte pluviométrico, el agua aplicada fluctuó entre $969 \mathrm{~m}^{3} \mathrm{ha}^{-1}$ en T1 y $1.575 \mathrm{~m}^{3} \mathrm{ha}^{-1}$ en T4 (Cuadro 3), constituyendo el riego un aporte suplementario en los meses de mayor evapotranspiración. Esto puede ser explicado por el corto período de riego de sólo 53 días y baja frecuencia de riego (7 días), constituyendo el riego un aporte suplementario a la precipitación. Hasta el momento, la investigación sobre requerimientos hídricos en Rosa Mosqueta es escasa, pero en cultivos resistentes a la sequía, resultados similares han sido obtenidos en Olivo (Olea europea L.) por Pérez-López et al. (2007) en una zona mediterránea de España con volúmenes aplicados en un rango de 610 a $830 \mathrm{~m}^{3} \mathrm{ha}^{-1}$. En esta investigación el mayor volumen se aplicó en T4 equivalente a $693 \mathrm{~m}^{3} \mathrm{ha}^{-1}$. Por su parte, Acevedo-Opazo et al. (2010) aplicaron volúmenes entre $111 \mathrm{~m}^{3} \mathrm{ha}^{-1}$ y $418 \mathrm{~m}^{3} \mathrm{ha}^{-1}$ en vid vinífera 'Cabernet Sauvignon', en Isla de Maipo, Chile. Estos bajos volúmenes hídricos aplicados en Rosa Mosqueta pueden ser explicados por el corto período de riego (53 días), baja frecuencia de riego (7 días), alta eficiencia del riego por goteo (90\%), alta capacidad de retención de agua $(0,18$ $\mathrm{m}^{3} \mathrm{~m}^{-3}$ ) del suelo franco arcilloso (Reynolds et al., 2009), y el aporte pluviométrico. Respecto de los cauadales, los goteros de $2 \mathrm{~L} \mathrm{~h}^{-1}$ (T2) y $4 \mathrm{~L} \mathrm{~h}^{-1}$ (T3) entregan una tasa de aplicación adecuada a la velocidad de infiltración del suelo y requerimientos hídricos del cultivo.

\section{Estado hídrico del suelo}

La evolución del contenido de agua del suelo a $30 \mathrm{~cm}$ de profundidad (Figura 1), permite determinar varia- ciones de la humedad durante el periodo de crecimiento lo que puede estar asociado con la alta demanda atmosférica, fenómeno que se puede observar en los tratamientos T1 y T2 los que estuvieron bajo el criterio de riego durante el mes de Enero. Por su parte, el T4 presentó los mayores niveles de humedad y en ciertos períodos resultó significativamente distinto a los demás tratamientos. Los tratamientos T3 y T4 se mantuvieron gran parte de la temporada entre capacidad de campo y criterio de riego, existiendo pequeñas variaciones entre ellos debido a los distintos volúmenes de agua aplicados. Durante el mes de Marzo se registró una baja en el porcentaje de humedad, debido a que el riego fue suspendido durante el mes anterior, lo que disminuyó el contenido de agua en el suelo. Sin embargo, los tratamientos mantuvieron contenidos similares de agua en el suelo, debido a la alta capacidad de retención de agua del suelo franco arcilloso y por el aporte pluviométrico en el período investigado $(88,2 \mathrm{~mm})$. Cabe destacar que durante el mes de Enero, los niveles de humedad de los tratamientos con 0,25 y 0,50 LR estuvieron cercanos a PMP, pero los valores de conductancia estomática (Figura 2) indican un nivel de transpiración adecuado de la planta. Esto destaca la necesidad de monitorear el nivel de humedad del suelo en el suelo y en la planta, por la diferente respuesta fisiológica de una especie ante el déficit hídrico. Además, Venegas (2014) determinó que la falta de riego durante el período de crecimiento del fruto (Enero) no afectó significativamente el rendimiento de Rosa Mosqueta.

\section{Conductancia estomática}

La conductancia estomática experimentó variaciones durante el período de crecimiento con valores iniciales entre 200 a 300 y finales entre 80 a 150 [mmol $\mathrm{m}^{-2} \mathrm{~s}^{-1}$, donde los tratamientos T1, T2 y T3 presentaron valores muy similares (Figura 2). En el mes de Enero fluctuaron entre 150-180 [mmol m$\left.{ }^{-2} \mathrm{~s}^{-1}\right]$ lo que significa que la transpiración fue adecuada al déficit de presión de vapor. Según Torres-Ruiz et al. (2013) valores bajo

Cuadro 3. Agua aplicada $\left(\mathrm{m}^{3} \mathrm{ha}^{-1}\right)$ con diferentes tratamientos hídricos en Rosa Mosqueta (Rosa canina L.) bajo riego por goteo en un suelo Aquic Palexeralfs.

Table 3. Water applied $\left(\mathrm{m}^{3} \mathrm{ha}^{-1}\right)$ to rose hip (Rosa canina L.) under drip irrigation with different hydric treatments in an Aquic Palexeralfs soil.

\begin{tabular}{|c|c|c|c|c|}
\hline Tratamientos hídricos & Q gotero $\left[\mathrm{L} \mathrm{h}^{-1}\right]$ & Riego $\left[\mathrm{m}^{3} \mathrm{ha}^{-1}\right]$ & $\mathrm{Pe}\left[\mathrm{m}^{3} \mathrm{ha}^{-1}\right]$ & Agua aplicada $\left[\mathrm{m}^{3} \mathrm{ha}^{-1}\right]$ \\
\hline T1: $0,25 \mathrm{LR}$ & 1 & 87 & 882 & 969 \\
\hline T2: $\quad 0,5$ LR & 2 & 173 & 882 & 1.055 \\
\hline T3: 1 LR & 4 & 347 & 882 & 1.229 \\
\hline T4: $2 \mathrm{LR}$ & 8 & 693 & 882 & 1.575 \\
\hline
\end{tabular}

LR: Lámina de riego aplicada en el predio; Q: Caudal; Pe: Precipitación efectiva. 


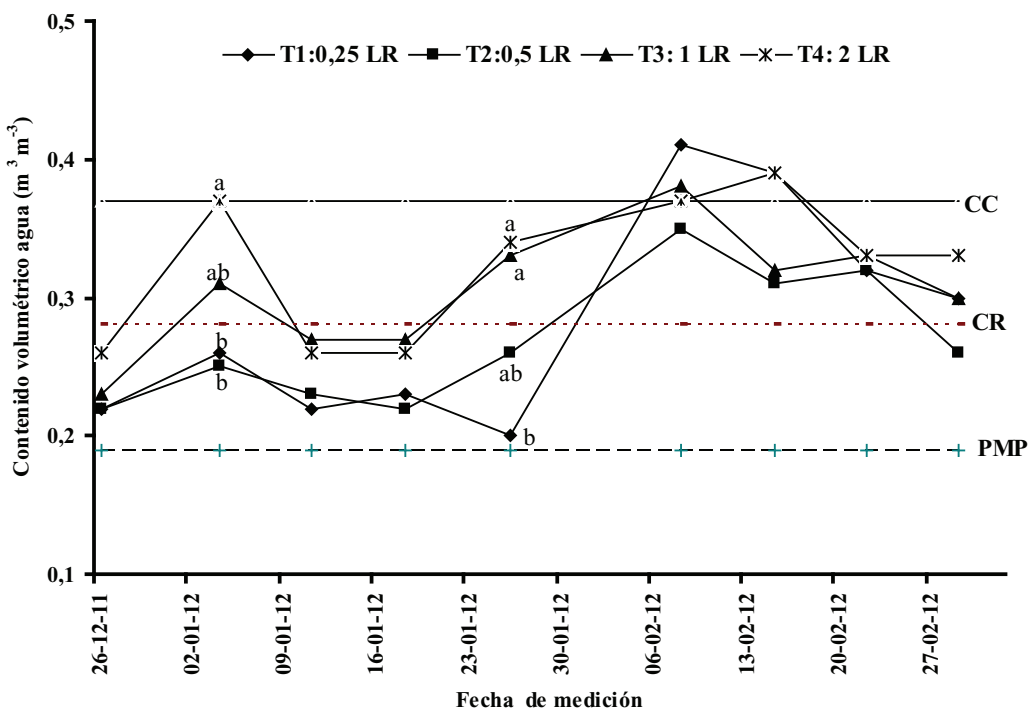

(*) Mediciones sin letras no presentan diferencias significativas (P > 0,05). LR: Lámina de riego ; CC: Capacidad de campo; CR: Criterio de riego; PMP: Punto de marchitez permanente.

Figura 1. Contenido agua del suelo $\left(\mathrm{m}^{3} \mathrm{~m}^{-3}\right)$ con diferentes tratamientos hídricos en Rosa Mosqueta (Rosa canina L.) bajo riego por goteo en un suelo Aquic Palexeralfs.

Figure 1. Soil water content $\left(\mathrm{m}^{3} \mathrm{~m}^{-3}\right)$ with different hydric treatments in rose hip (Rosa canina L.) under drip irrigation in an Aquic Palexeralfs soil.

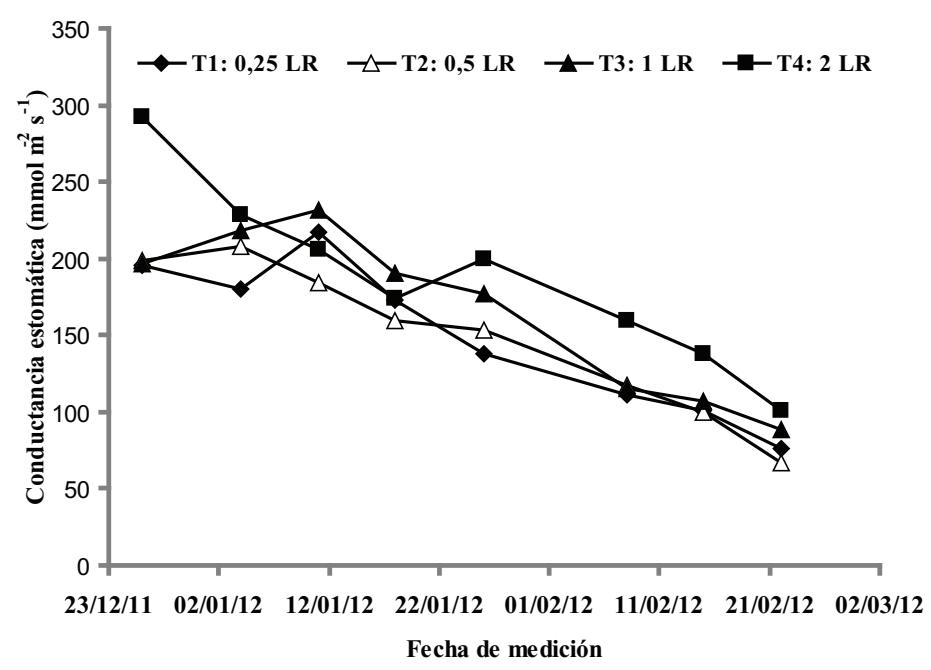

Figura 2. Conductancia estomática $\left[\mathrm{mmol} \mathrm{m}^{-2} \mathrm{~s}^{-1}\right]$ con diferentes tratamientos hídricos en Rosa Mosqueta (Rosa canina L.) bajo riego por goteo en un suelo Aquic Palexeralfs.

Figure 2. Stomatal conductance [ $\left.\mathrm{mmol} \mathrm{m}^{-2} \mathrm{~s}^{-1}\right]$ with different hydric treatments in rose hip (Rosa canina L.) under drip irrigation in an Aquic Palexeralfs soil.

$100\left[\mathrm{mmol} \mathrm{m}^{-2} \mathrm{~s}^{-1}\right]$ significan estrés hídrico severo asociado con humedad aprovechable (HA) del suelo muy inferior al criterio de riego (50\% HA) y potencial hídrico xilemático de -3,5 a -4,5 MPa. Respecto de la temporada de crecimiento, la difusión del vapor de agua fue disminuyendo, lo que se explica por la mayor demanda hídrica de la planta en etapas reproductivas como cuaja y/o llenado del fruto (Diciembre-Enero). Cabe mencionar que la conductancia estomática no sólo es afectada por la disponibilidad hídrica, sino que también por factores medioambientales como iluminación, velocidad del viento, estado de la hoja y por la heterogeneidad de la distribución del agua en la zona radical (Quezada et al., 2005; Torres-Ruiz et al., 2013). 


\section{Componentes del rendimiento y rendimiento}

El número de frutos por planta y la producción por planta presentaron diferencias significativas $(\mathrm{P} \leq 0,05)$ con los diferentes niveles de agua aplicados (Cuadro 4). El tratamiento T3 con goteros de $4 \mathrm{~L} \mathrm{~h}^{-1}$ mostró un mayor número de frutos y una mayor producción por planta, esto significa que con la lámina de riego aplicada en el predio, la planta expresa su potencial productivo debido a que en la zona de mayor concentración de raíces existe una buena relación agua: aire, con una porosidad total de $52,9 \%$ en la profundidad de $0-28$ $\mathrm{cm}$. Esto concuerda con Bravo (1995), quien señala que el mayor rendimiento se puede atribuir al mayor número de frutos por planta pero no al peso promedio de los frutos, ya que al producir más frutos el peso de los mismos disminuye. Es importante mencionar que, en general, se observa que una restricción o exceso hídrico se traduce en un menor número de frutos cuajados sin manifestar un deterioro en el peso de éstos, lo que coincide con lo determinado en frambuesa por Holzapfel et al. (1994).

El diámetro longitudinal y ecuatorial de los frutos en los distintos tratamientos hídricos (Cuadro 4) no presentó diferencias significativas $(\mathrm{P}>0,05)$, lo que concuerda con Nissen et al. (2010) quienes determinaron que en murtilla (Ugni molinae Turcz.) el diámetro del fruto no presentó diferencias significativas en función de la dosis de riego. En relación al peso promedio de los frutos (Cuadro 4) estos no presentaron diferencias significativas $(\mathrm{P}>0,05)$, por lo que se puede inferir que los niveles de riego aplicados no tuvieron efecto sobre esta propiedad. Esto no concuerda con Bravo (1995) quienes en un estudio realizado en frambuesa (Rubus idaeus L.) señalan que el peso promedio de los frutos se eleva a medida que aumenta los niveles de reposición hídrica en las etapas de mayor demanda.

\section{Rendimiento industrial}

El rendimiento industrial no presentó diferencias significativas $(\mathrm{P}>0,05)$ con los tratamientos hídricos aplicados (Cuadro 4). La mayor conversión de kg de fruto fresco a kg de pulpa seca muestra una tendencia a ser más eficiente en los tratamientos con menor lámina de riego aplicada, entre $3,1 \mathrm{~kg}$ con goteros de 4 $\mathrm{L} \mathrm{h}^{-1}$ (1 LR) y 3,2 kg con goteros de $2 \mathrm{~L} \mathrm{~h}^{-1}(0,25 \mathrm{LR})$ en comparación a 3,3 $\mathrm{kg}$ con goteros de $8 \mathrm{~L} \mathrm{~h}^{-1}$ (2LR). Según Aghamirov y Farzaliev (2005) Rosa canina L. es una especie de bajos requerimientos hídricos, resistente a la sequía y altas temperaturas, por lo cual el riego en zonas de secano puede considerarse como un aporte suplementario al agua lluvia, para su crecimiento y desarrollo. Los rendimientos son similares a los obtenidos por Hevia et al. (1999) quienes determinaron que la cantidad de pulpa seca está en relación directa con la cantidad de agua que contiene el fruto. Es importante destacar que, de acuerdo con los resultados obtenidos en el rendimiento de pulpa seca, el tratamiento con 0,5 LR no presentó diferencias significativas $(P>0,05)$ con los tratamientos con mayores niveles de riego. Por lo tanto, se puede concluir que el tratamiento T2 con la mitad de la lámina de riego aplicada en el predio es una estrategia de riego adecuada para la producción de pulpa seca en zonas con escasez de recursos hídricos, ya que no presentó diferencias significativas $(\mathrm{P}>0,05)$ con el tratamiento control (T3).

\section{Productividad del agua}

La productividad del agua en Rosa canina L. disminuye con el volumen de agua aplicada como riego suplementario (Cuadro 5) desde $86,6 \mathrm{~kg} \mathrm{~m}^{-3}$ con $0,25 \mathrm{LR}$ a $13,9 \mathrm{~kg} \mathrm{~m}^{-3}$ con $2 \mathrm{LR}$, en cambio con el total de agua aportada (riego + lluvia) aumenta desde 7,8 $\mathrm{kg} \mathrm{m}^{-3}$ con

Cuadro 4. Componentes de rendimiento y rendimiento industrial para diferentes tratamientos hídricos en Rosa Mosqueta (Rosa canina L.) bajo riego por goteo en un suelo Aquic Palexeralfs.

Table 4. Yield components and industrial yield with different hydric treatments in rose hip (Rosa canina L.) under drip irrigation in an Aquic Palexeralfs soil.

\begin{tabular}{cccccccc}
\hline Tratamientos hídricos & NFP & PPF & DL & DE & PPL & RI & RPS \\
\hline T1: $0,25 \mathrm{LR}$ & $1.154 \mathrm{~b}$ & $218,0 \mathrm{a}$ & $2,0 \mathrm{a}$ & $1,5 \mathrm{a}$ & $2,3 \mathrm{c}$ & $3,2 \mathrm{a}$ & $2.379,5 \mathrm{c}$ \\
T2: $0,5 \mathrm{LR}$ & $1.478 \mathrm{ab}$ & $198,9 \mathrm{a}$ & $1,9 \mathrm{a}$ & $1,4 \mathrm{a}$ & $3,1 \mathrm{~b}$ & $3,2 \mathrm{a}$ & $3.284,1 \mathrm{ab}$ \\
T3: 1 LR & $1.804 \mathrm{a}$ & $211,9 \mathrm{a}$ & $2,0 \mathrm{a}$ & $1,4 \mathrm{a}$ & $3,9 \mathrm{a}$ & $3,1 \mathrm{a}$ & $3.912,7 \mathrm{a}$ \\
T4: 2 LR & $1.395 \mathrm{ab}$ & $230,5 \mathrm{a}$ & $2,0 \mathrm{a}$ & $1,4 \mathrm{a}$ & $2,9 \mathrm{~b}$ & $3,3 \mathrm{a}$ & $3.020,3 \mathrm{bc}$ \\
\hline CV \% & 12,9 & 13,1 & 4,5 & 4,3 & 5,3 & 3,1 & $3,1 \mathrm{x}$ \\
\hline
\end{tabular}

LR: Lámina de riego; NFP: Número de frutos por planta; PPF: Peso promedio frutos por planta (g); DL: Diámetro longitudinal (cm); DE: Diámetro ecuatorial (cm); PPL: Producción por planta (kg); RL: Rendimiento industrial (kg fruto fresco $\mathrm{kg}^{-1}$ pulpa seca); RPS: Rendimiento de pulpa seca ( $\mathrm{kg} \mathrm{ha}^{-1}$ ); CV: Coeficiente de variación. 
Cuadro 5. Productividad del agua con diferentes tratamientos hídricos en Rosa Mosqueta (Rosa canina L.) bajo riego por goteo en un suelo Aquic Palexeralfs.

Table 5. Water productivity with different hydric treatments in rose hip (Rosa canina L.) under drip irrigation in an Aquic Palexeralfs soil.

\begin{tabular}{lcccc}
\hline Tratamientos hídricos & Rendimiento $\left[\mathrm{kg} \mathrm{ha}^{-1}\right]$ & PRS $\left[\mathrm{kg} \mathrm{m}^{-3}\right]$ & PAA $\left[\mathrm{kg} \mathrm{m}^{-3}\right]$ & $\Delta \mathrm{R} / \Delta \mathrm{Q}\left[\mathrm{kg} \mathrm{m}^{-3}\right]$ \\
\hline T1: $0,25 \mathrm{LR}$ & $7.533 \mathrm{c}$ & $86,6 \mathrm{a}$ & $7,8 \mathrm{a}$ & - \\
T2: 0,5 L R & $10.265 \mathrm{~b}$ & $59,3 \mathrm{~b}$ & $97 \mathrm{~b}$ & 31,8 \\
T3: 1 LR & $12.888 \mathrm{a}$ & $37,1 \mathrm{c}$ & $10,5 \mathrm{c}$ & 15,1 \\
T4: $\quad 2$ LR & $9.643 \mathrm{~b}$ & $13,9 \mathrm{~d}$ & $6,1 \mathrm{~d}$ & $-9,4$ \\
\hline
\end{tabular}

LR: Lámina riego; PRS: Productividad del riego suplementario; PAA: Productividad agua aportada; $\Delta \mathrm{R} / \Delta \mathrm{Q}: \Delta$ Rendimiento / $\Delta$ Agua aplicada.

$0,25 \mathrm{LR}$ a $10,5 \mathrm{~kg} \mathrm{~m}^{-3}$ con 1 LR para luego disminuir a $6,1 \mathrm{~kg} \mathrm{~m}^{-3}$ (T4). Al respecto, Bravo (1995), determinó en un estudio realizado en frambuesa que existe una relación inversa entre la cantidad de agua aplicada y productividad del agua. En cuanto a la relación del incremento marginal de rendimiento y agua se puede apreciar que el T2 (0,5 LR) presentó el valor más alto $\left(31,8 \mathrm{~kg} \mathrm{~m}^{-3}\right)$ lo que comprueba que Rosa canina L. es un arbusto con bajos requerimientos hídricos. Al respecto, Schmidt (1992), señala que sería posible lograr los mayores aumentos marginales al someter a la planta a un déficit hídrico, pero con una disminución del rendimiento final. Cabe mencionar, que el tratamiento de mayor reposición hídrica (2 LR) obtuvo el valor más bajo en PA y también la relación rendimiento y agua fue negativa $\left[-9,4 \mathrm{~kg} \mathrm{~m}^{-3}\right]$, lo que indica que el consumo de agua por parte del frutal llegó a su máximo y volúmenes de agua excesivos causan una disminución del rendimiento por saturación del suelo y asfixia radical, lo que concuerda con lo reportado por Quezada et al. (2011) en una investigación realizada en manzanos.

\section{CONCLUSIONES}

Los requerimientos hídricos de Rosa Mosqueta para la zona de Chillán fueron bajos entre 173 y 347 $\mathrm{m}^{3} \mathrm{ha}^{-1} \mathrm{y}$ correspondieron a un riego suplementario en el período de mayor evapotranspiración. La aplicación de diferentes niveles de riego no afectaron significativamente los parámetros fisiológicos de la planta ni los componentes de rendimiento. La aplicación del 50\% de la lámina de riego con goteros de $2 \mathrm{~L} \mathrm{~h}^{-1}$ es una estrategia de riego adecuada para la producción de pulpa seca en zonas con escasez de recursos hídricos.

\section{REFERENCIAS}

ACEVEDO-OPAZO, C., ORTEGA-FARIAS, S., FUENTES, S. 2010. Effects of grapevine (Vitis vinifera L.) water status on water consumption, vegetative growth and grape quali- ty: an irrigation scheduling application to achieve regulated deficit irrigation. Agricultural Water Management. 97: 956-964.

AGHAMIROV, U.M., FARZALIEV, V.S. 2005. Evaluation of five introduced rose species in Azerbaijan. Acta Horticulture 690: 49-55.

ALARCÓN, J.J., ORTUÑO, M.F., NICOLÁS, E., NAVARRO, A., TORRECILLAS, A. 2006. Improving water-use efficiency of young lemon trees by shading with aluminised-plastic nets. Agricultural Water Management 82: 387-398.

BERTI, M., JOUBLAN, J. 1997. Rosa mosqueta: una nueva alternativa agrícola para zonas de secano. Agroanálisis 156: 29-33.

BILLIB, M., HOLZAPFEL, E., FERNÁNDEZ-CIRELLI, A. 2009. Sustainable water resources management for irrigated agriculture in Latin America. Chilean Journal Agricultural Research 69: 3-5.

BRAVO, M. 1995. Efecto de dos métodos de riego y volúmenes diferenciales de agua, sobre el desarrollo y producción de frambuesos rojos (Rubus idaeus L.) cv. Heritage de tercer año. Tesis Ingeniero Agrónomo. Universidad de Concepción, Chillán, Chile. 105 p.

DEL POZO, A., DEL CANTO, P. 1999. Áreas agroclimáticas y sistemas productivos en la VII y VIII regiones. Serie Quilamapu N 113. INIA Quilamapu. Chillán.

GÜNES, M. 2010. Pomological and phenological characteristics of promising rose hip (Rosa) genotypes. African Journal of Biotechnology 9: 6301-6306.

HEVIA, F., JOUBLAN, J.P., BERTI, M., TELLO, M., GALAZ, A., WILCKENS, R. 1999. Fecha de cosecha de rosa mosqueta: calidad y rendimiento de cascarilla. Agro-Ciencia 15: 285-289.

HOLZAPFEL, E., HEPP, R., SCHMIDT, S., MATTA, M. 1994. Efecto del nivel de agua aplicado y la frecuencia del riego 
en el desarrollo y producción de frambuesos de un año. Agro-Ciencia 10: 51-56.

INFOSTAT. 2004. Infostat: Software estadístico. Manual del usuario. Versión 2004.Grupo Infostat. FCA, Universidad Nacional de Córdoba. Editorial Brujas, Córdoba.

JOUBLAN, J.J., RÍOS, D. 2005. Rose culture and industry in Chile. Acta Horticulturae 690: 65-69.

KOVACS, S., FACSAR,G., UDVARDY, L., TOTH, M. 2005. Phenological, morphological an pomological characteristics of some rose species found Hungary. Acta Horticulturae 690: 71-76.

MEDEL, F. 1979. Prospección de arbustos frutales en el sur de Chile. Agro Sur 7: 94-97.

NISSEN, J., DEL RÍO, M., SEGUEL, I., TORRALBO, L. 2010. Efecto de diferentes manejos hídricos sobre el crecimiento vegetativo y reproductivo de murtilla (Ugni molinae Turcz.). Agro Sur 38: 42-54.

OBANDO, L., Mc LEOD, C. 2009. Establecimiento y mantención de huertos de zarzaparrillas y grosellas para Magallanes: antecedentes generales. Boletín INIA. Nㅜ 201. INIA Kampenaike. Punta Arenas.

OWEIS, T., ZHANG, H., PALA, M. 2000. Water use efficiency of rainfed and irrigated bread wheat in a Mediterranean environment. Agronomy Journal 92: 231-238.

PÉREZ-LÓPEZ, D., RIBAS, F., MORIANA, A., OLMEDILLA, N., DE JUAN, A. 2007. The effect of irrigation schedules on the water relations and growth of a young olive (Olea europaea L.) orchard. Agricultural Water Management 89: 297-304.

QUEZADA, C., RIQUELME, F., GARCÍA, H. 2005. La resistencia difusiva como indicador del estado hídrico de la planta. Ciencia Ahora 8: 90-95.

PÉREZ-LÓPEZ, D., RIBAS, F., MORIANA, A., OLMEDILLA, N., DE JUAN, A. 2007. The effect of irrigation schedules on the water relations and growth of a young olive (Olea europaea L.) orchard. Agricultural Water Management. 89: 297-304.

QUEZADA, C., SOLÍS, E., VENEGAS, A., FAÚNDEZ, M. 2011. Efecto de cuatro niveles de aplicación de agua en rendimiento y calidad de un huerto de manzano (Malus do- mestica Borkh.) "Fujï bajo riego por goteo. Agro-Ciencia 27: 65-75.

REYNOLDS, W.D., DRURY, C.F, TAN, C.S., FOX, C.A., YANG, X.M. 2009. Use of indicators and pore volumen-function characteristics to quantify soil physical quality. Geoderma 152: $252-263$

SANDOVAL, M., DÖRNER, J., SEGUEL, O., CUEVAS, J., RIVERA, D. 2012. Métodos de análisis físicos de suelos. Publicaciones Departamento de Suelos y Recursos Naturales $\mathrm{N}^{\circ}$ 5. Universidad de Concepción. Facultad de Agronomía. Chillán.

SCHMIDT, S. 1992. Efecto de la frecuencia de riego y diferentes niveles de agua sobre el desarrollo y producción de frambuesas (Rubus idaeus L.) cv. Heritage. Tesis Ingeniero Agrónomo. Universidad de Concepción, Chillán, Chile. $103 \mathrm{p}$.

STOLPE, N. 2006. Descripciones de los principales suelos de la VIII Región de Chile. Publicaciones Departamento de Suelos y Recursos Naturales $\mathrm{N}^{\circ} 1$. Universidad de Concepción. Facultad de Agronomía. Chillán.

SUDZUKI, F. 1997. Cultivo de frutales menores. (6a. ed.). Universitaria. Santiago.

TORRES-RUIZ, J.M., DIAZ-ESPEJO, A., MORALES-SILLERO, A., MARTÍN-PALOMO, M.J., MAYR, S., BEIKIRCHER, B., FERNÁNDEZ, J.E. 2013. Shoot hydraulic characteristics, plant water status and stomatal response in olive trees under different soil water conditions. Plant and Soil 373: 77-87.

UGGLA, N., MARTINSSON, M. 2005. Cultivate the wild rosesexperiences from rose hip production in Sweden. Acta Horticulturae 690: 83-89.

VARBLE, J.L, CHÁVEZ, J.L. 2011. Performance evaluation and calibration of soil water content and potencial sensors for agricultural soils in eastern Colorado. Agricultural Water Management 101: 93-106.

VENEGAS, R. 2014. Riego deficitario controlado en Rosa Mosqueta (Rosa canina L.).Tesis Ingeniero Agrónomo. Universidad de Concepción. 24 p.

VITA, F., LIOTTA, M., PARERA, C. 2004. Effects of irrigation deficit on table grape cv. Superior Seedless production. Acta Horticulturae 646: 183-186. 
